\title{
DEPRESSIVE DISORDERS IN EPILEPSY
}

\author{
K. Todorova ${ }^{1}$, M. Arnaoudova ${ }^{2}$ \\ 1) First Psychiatric Clinic, MHAT "St. Marina", \\ 2) Third Psychiatric Clinic, MHAT "St. Marina", \\ Department of Psychiatry, \\ Medical University, Varna, Bulgaria
}

\section{SUMMARY:}

Depressive disorders are the most frequent psychiatric comorbidity in epilepsy but very often remain unrecognized and untreated. We examined 103 epileptic patients, aged 18-60 years, 40 males and 63 females, for the presence of interictal depressive disorder. All subjects underwent clinical psychiatric examination, including evaluation on Hamilton Depression Rating Scale (HAM-D17). A questionnaire for demographic and seizure-related variables was also completed. Concurrent depressive disorder (clinically presented according to ICD-10 diagnostic criteria) affected $28.3 \%$ of all evaluated patients. Based on HAM-D-17 scores depression was defined as mild - $80 \%$ of all depressed patients, moderate $-17 \%$ and severe $-3 \%$. Atypical presentation of interictal depressive disorder was frequent. Depression has a tremendous effect on one's family, social and psychological functioning, even more than the actual seizure frequency and severity. Diagnostic difficulties come through the atypical mode of presentation of depressive disorders in epilepsy. Proper neuropsychiatric evaluation is essential for improving treatment and quality of life for patients with epilepsy.

Key words: Epilepsy, Depressive Disorders, Quality of life

\section{INTRODUCTION:}

In patients with epilepsy, mood disorders represent a frequent psychiatric comorbidity. Depressive disorders lead the list with the prevalence of interictal depressive disorder in patients with epilepsy shown to range between 9 and 55\% depending on the sample population and the methods of assessment (12). By contrast the prevalence of depression in the general population is reported to be $1-3 \%$ in men and 2-9\% in women (8). A bidirectional relationship between epilepsy and depression is supposed and studies suggest that depression and epilepsy may share common pathogenic mechanisms and the occurrence of one may facilitate the development of the other and vice versa (9, 11). Multiple factors are implicated in the development of depression in epilepsy including clinical (seizure frequency, seizure type or foci, epilepsy duration, age at onset, antiepileptic drugs) and psychosocial ones (life stressors, employment, marital status, quality of life)(13).

Controversy exists regarding the phenomenology of depression in epilepsy. According to many authors interictal depressive disorder in epileptic patients most commonly represents as a pleomorphic cluster of depressive symptoms characterized by fewer neurotic traits, higher trait and state of anxiety, most often related to seizures, high irritability, explosive anger and somatic symptoms as pain, disturbed sleep, decreased energy, tiredness and fatigue $(1,11,12)$. Although the overall severity of these depressive disorders is usually mild, they cause significant disruption to patients' daily activities, social relations and quality of life (11).

\section{AIM:}

The aim of our current study is to assess the frequency and some aspects of the phenomenology of interictal depressive disorder in a random sample of adult patients with idiopatic epilepsy.

\section{SUBJECTS AND METHODS:}

We examined 103 consequative patients, 40 males and 63 females, with idiopatic epilepsy, aged between $18-60$, without any other somatic or neurological comorbidity at the time of the psychiatric evaluation. The duration of epilepsy had to be medically proven for more than 12 months and patients had to be seizure-free for the last 72 hours before entering the study. All subjects underwent clinical psychiatric examination, including evaluation on Hamilton Depression Rating Scale (HAM-D-17) (7). A specially designed questionnaire for demographic and seizure-related variables was also completed.

\section{RESULTS:}

Concurrent depressive disorder (clinically presented and according to ICD-10 diagnostic criteria) affected 30 (28.3\%) of all evaluated patients.

Based on HAM-D-17 scores the severity of the depressive disorder was defined as mild (7-17 points) - 24 ( $80 \%$ ) patients, moderate (18-24 points) -5 (17\%) patients and severe (>24 points) - 1 (3\%) patient (Fig. 1).

Among all depressed epilepsy patients 18 (60\%) had partial seizures, accompanied or not by secondary 
generalization (according to ILAE classification) and $12(40 \%)$ had primarily generalized seizures (9). Seizure free were 3 $(10 \%)$ of all depressed subjects, $7(23 \%)$ had less than one seizure/month and $20(67 \%)$ had seizure frequency $>1 /$ month.

Atypical presentation of interictal depressive disorder was frequent and yet some typical features for the depressive disorder in our patient group should be pointed out Overestimated ideas of worthlessness were reported by 12 $(40 \%)$ of all evaluated patients and another 11 (36.7\%) reported overestimated ideas of guilt (Fig. 2). Feelings life is not worth living reported $11(37.9 \%)$ of all depressed patients, wishes to be dead $-3(10.3 \%)$ and suicidal ideas or gestures shared $3(10.3 \%)$ of all depressed patients (Fig. 3).

In keeping with others we found that decrease in energy level and productivity, fatigue or weakness were closely related to the comorbid depression in epilepsy and a dissociation was detected between the high intensity of these symptoms and the predominating mild severity of the interictal depressive disorder (6).

Thoughts and feelings of incapacity, fatigue or weakness related to activities, work or hobbies reported 7 $(23.3 \%)$ of all depressed patients, loss of interest in activity, hobbies or work was present in $8(26.7 \%)$ and decrease in actual time spent in activities or decrease in productivity reported 10 (33.3\%) of all depressed epilepsy patients(Fig. 4).

Psychomotor retardation, based on observation of slowness of thought and speech, impaired ability to concentrate, decreased motor activity was present in the depressed epilepsy group and the comorbidity of epilepsy and depression probably augmented the frequency and severity of the symptom.

Comorbid depressive disorder and anxiety, wheather or not related to seizures, are closely associated in epilepsy. Comorbid depressive disorder usually augments interictal anxiety and the detection of high state of anxiety related to seizures should raise questions about presence of comorbid depression (3).

High state of psychic anxiety based on HAM-D-17 scores reported 24 (80\%) of all depressed epilepsy patients. Subjective tension and irritability were present in $9(30 \%)$ of the patients with comorbid depressive disorder, $12(40 \%)$ were worrying about minor matters and $3(10 \%)$ showed apprehensive attitude apparent in face or speech (Fig. 5).

Somatic anxiety presented by psysiological concomitants of anxiety: gastro-intestinal, cardio-vascular, respiratory, urinary symptoms, etc. was detected in 23 $(79.3 \%)$ of the epilepsy patients with comorbid depressive disorder.

\section{DISCUSSION:}

The results of the present study confirm the assumption that patients with epilepsy are at high risk of depression. High rates of comorbidity may be due to a shared pathophysiological mechanism between epilepsy and depression, however coincidence or selection bias cannot always be excluded. In addition, genetic, psychosocial, and iatrogenic factors may also contribute to this finding (2). Controversy exists over the distinct and unique forms of psychopathology in epilepsy including the phenomenology of depression. Neither traditional systems of classification used in psychiatry, such as the DSM or the ICD, which club the disorders under the broad umbrella of organic mental disorders, not the ILAE classification, which does not address the psychiatric components of the disorder do justice to these "epilepsy-specific" syndromes. Regarding interictal depressive disorder, Blumer et al. (2000) coined the term interictal dysphoric disorder and Kanner et al. (2002) referred to it as dysthymic-like disorder of epilepsy $(4,11)$. Our data is in consistency with the atypical mode of presentation of interictal depressive disorder in epilepsy which makes the diagnosis of depressive disorders in epilepsy population sometimes difficult. In keeping with other data that epilepsy patients tend to minimize their psychiatric symptoms most of our patients reported the depressive symptoms when actively inquired for their presence rather than spontaneously because of fear of being further stigmatized or trying to consider them as a reflection of a "normal adaptation process to the epilepsy itself" (10, $11)$.

\section{CONCLUSION:}

Comorbid depressive disorder in epilepsy is relatively common and no evaluation of these patients is complete without investigation of a prior or concurrent depressive symptoms. Advances in understanding the psychiatric comorbidity in epilepsy move the treatment of patients toward a comprehensive biopsychosocial model that focuses on the whole person rather than simply on the disease process which is in favour of improving the Quality-of-Life of these patients.

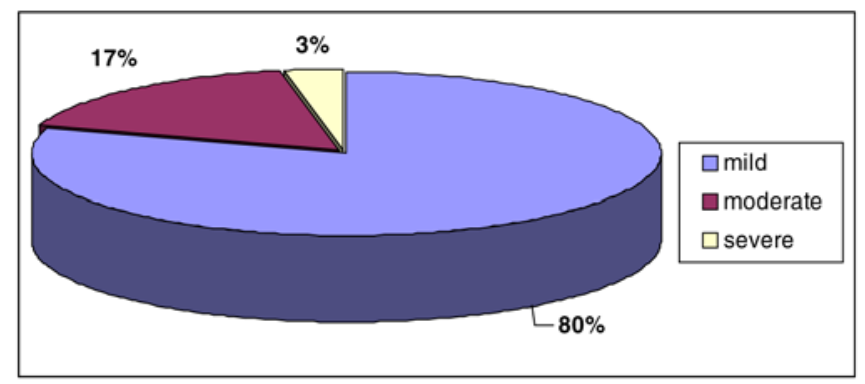

Fig. 1. Severity of the Comorbid Depressive Disorder based on HAM-D-17 scores. 


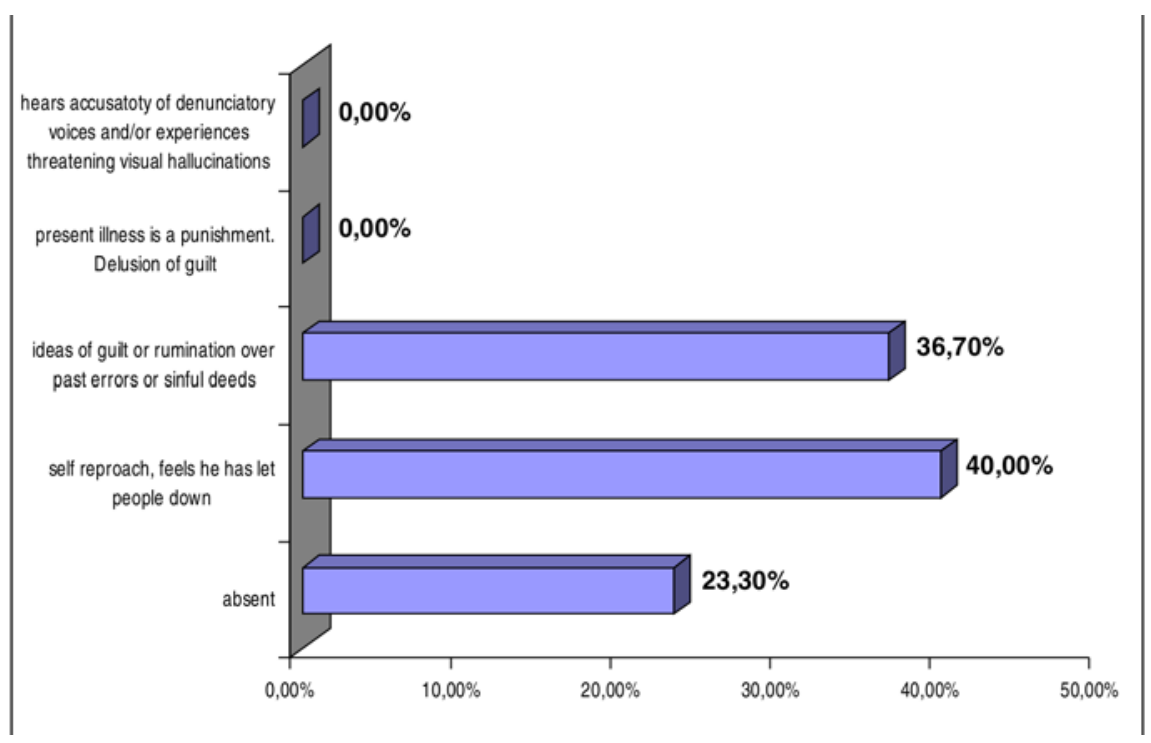

Fig. 2. Feelings of guilt according to HAM-D-17 scores.

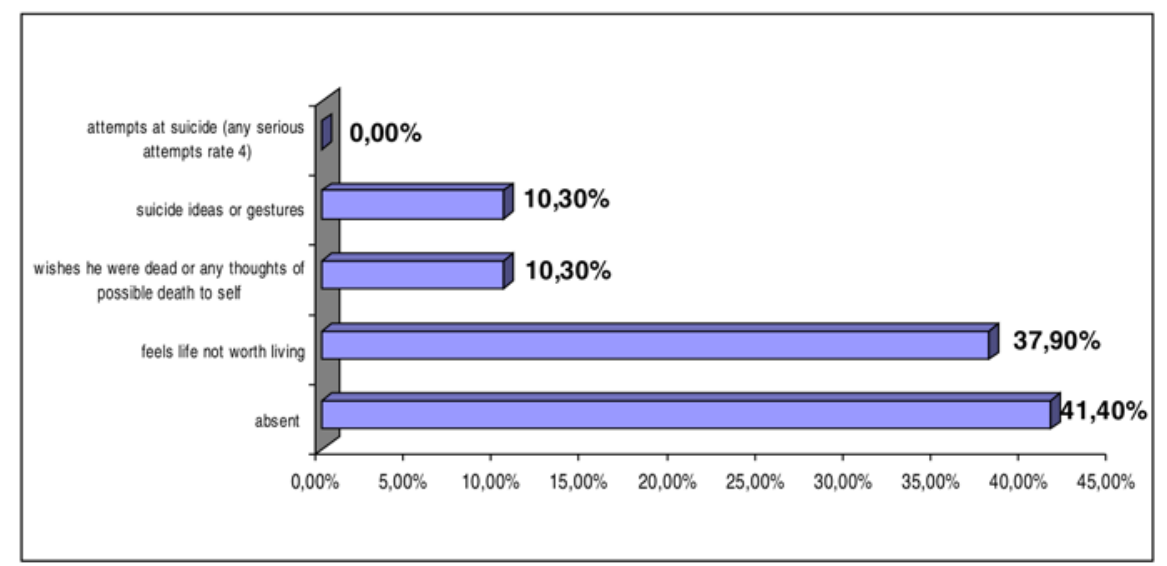

Fig. 3. Suicide according to HAM-D-17 scores.

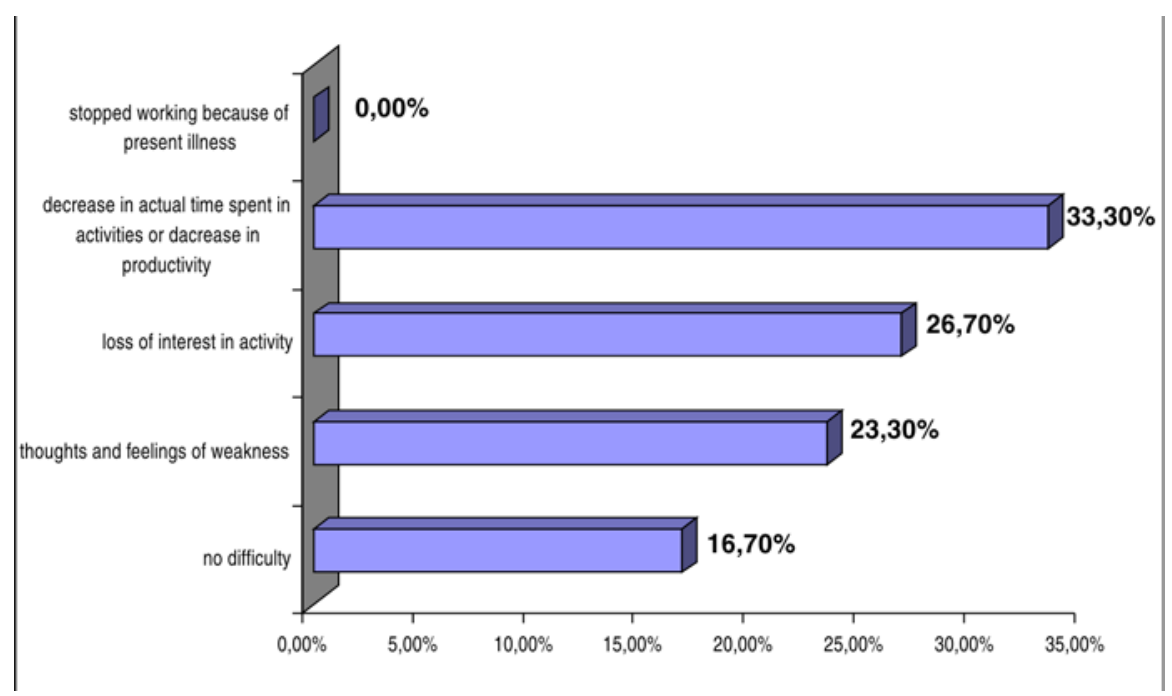

Fig. 4. Work and activities according to HAM-D-17 scores. 


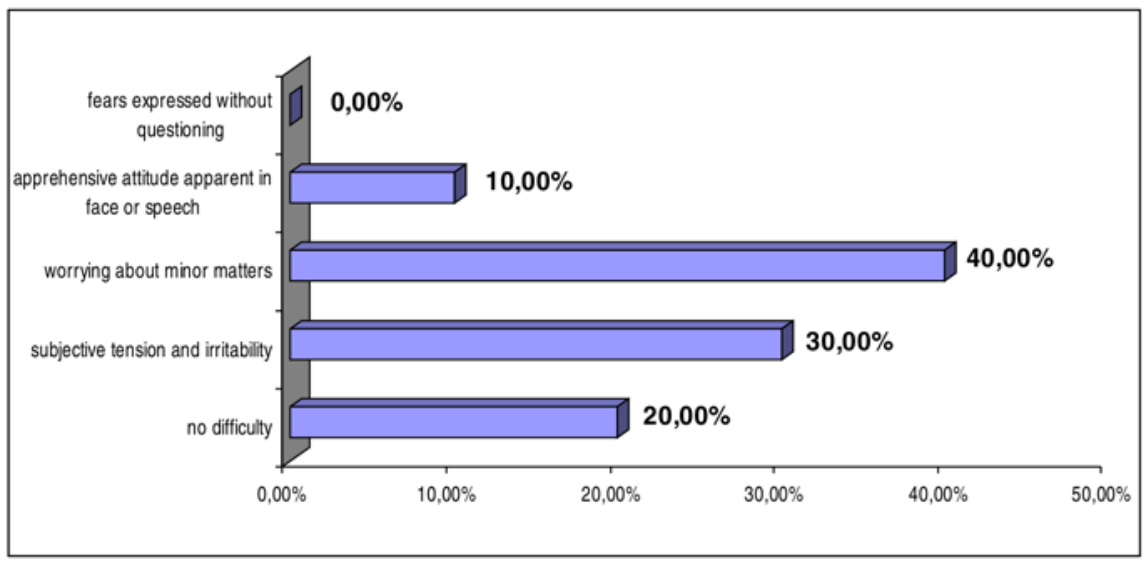

Fig. 5. Psychic anxiety according to HAM-D-17 scores.

\section{REFERENCES:}

1. Beghi, E., Roncolato, M., Visona, G. Depression and altered quality of life in women with epilepsy of childbearing age. Epilepsia, 45, 2004, 1, 64-70.

2. Beyenburg, S., Cristian D. Psychiatric comorbidity in epilepsy. Bull Soc Sci Med Grand Duche Luxemb, 3, 2005, 238-292.

3. Beyenburg, S. et al. Anxiety in patients with epilepsy: Systematic review and suggestions for clinical management. Epilepsy Behav, 7, 2005, 161-171.

4. Blumer D. Dysphoric disorders and paroxysmal affects: Recognition and treatment of epilepsy-related psychiatric disorders. Harv Rev Psychiatry, 2000, May-Jun;8(1):8-17.

5. Comission on Classification and
Terminology of the International League Against Epilepsy. Proposal for revised classification of epilepsies and epileptic syndromes. Epilepsia, 30, 1989, 389-399.

6. Ettinger, A. et al. Fatigue and depression in epilepsy. Journal of epilepsy, 1998; 11(2): p105-109.

7. Hamilton M. A rating scale for depression. J Neurol Neusurg Psychiatry 1960; Feb; 23:56-62.

8. Harden, C., Goldstein, M. Mood disorders in patients with epilepsy: epidemiology and management. CNS Drugs, 16, 2002, 291-302.

9. Hesdorffer DC, Hauser WA, Olafsson E, Ludvigsson P, Kjartansson O. Depresson and suicide attempt as risk factors for incident unprovoked seizures.
Ann Neurol. 2006 Jan;59(1):35-41.

10. Kanner AM. Depression in epilepsy: a complex relation with unexpected consequences. Curr Opin Neurol. 2008, Apr;21(2):190-194.

11. Kanner, A., Balabanov, A. Depression and epilepsy: how closely related are they? Neurology, 58, 2002, Supp1.5,S27-S39.

12. Lambert, M., Robertson, M. Depression in epilepsy: etiology, phenomenology, and treatment. Epilepsia 40, 1999, Suppl.10, S21-47.

13. Titlic M, Basic S, Hajnsek S, Lusic I. Comorbidity psychiatric disorders in epilepsy: a review of literature. Bratisl Lek Listy. 2009;110(2):105-109.

\author{
Address for correspondence: \\ Koralia Todorova, MD \\ First Psychiatric Clinic, MHAT "Sveta Marina" \\ 1, Hristo Smirnenski str, 9010 Varna, Bulgaria \\ Mobile: $+359 / 888376327$ \\ E-mail: koralia_todorova@yahoo.com
}

